\title{
Frecuencia de pacientes ingresados por intentos suicidas en el servicio de salud mental del Hospital General Tercer Milenio, Aguascalientes
}

García-Huizar María del Pilar*, Antúnez-Salinas L**, Gutiérrez-Mendoza Israel***,

Góngora-Ortega Javier***

\section{Resumen}

- Objetivo: Establecer la frecuencia y características psico- sociales en pacientes hospitalizados con intento suicida - en el servicio de salud mental del Hospital General Ter-

- cer Milenio (HGTM). Material y métodos: Se realizó un

- estudio observacional, transversal, descriptivo y retros-

- pectivo; en pacientes hospitalizados en el servicio de

- Salud Mental del Hospital General Tercer Milenio, por

- presentar intento de suicidio en pacientes de cualquier

- edad y sexo. Se recabaron las variables psicosociales

- obtenidas del expediente clínico por medio de una cé-

- dula de recolección de datos. Se aplicó la prueba x2

mediante el paquete estadístico SPSS 15.0. Resultados:

- El número de pacientes atendidos por intentos suicidas

- fue de 94, que corresponde al 38.8\% de los ingresos en el Servicio de Salud Mental, predominó el sexo femenino con 63\%, edad $25.4 \pm 11$. I años, $67 \%$ solteros, $42 \%$ recibían tratamiento psiquiátrico, $45 \%$ tenían antecedente de intento suicida, el mecanismo de lesión actual más frecuente fue intoxicación medicamentosa (69\%). El motivo más frecuente fue problemas con su pareja (28\%), el trastorno depresivo fue la comorbilidad predominante (20\%), el $21 \%$ de los pacientes usaban drogas; el mecanismo de lesión más frecuente fue la intoxicación medicamentosa para el femenino y el ahorcamiento en el masculino ( $p<0.00 \mathrm{I}$ ).

Conclusión: La prevalencia de intentos suicidas fue de el 38.8\% de los pacientes hospitalizados en el servicio de Salud Mental del HGTM. LUXMÉDICA, AÑO 7 NÚM 21 MAYO-AGOSTO 2012 pp 9-16

Palabras clave: Intento suicida, prevalencia, salud mental, hospital general.

* Médico Psiquiatra del Hospital General Tercer Milenio.

** Médico Integrista del Hospital General Tercer Milenio

*** Investigadores de la Secretaría de Salud de Aguascalientes.

Fecha de recibido: 2 abril 2012

Fecha de aceptación: 29 de junio 2012

Correspondencia: Dra. María del Pilar García Huizar: Servicio de Salud Mental del Hospital General Tercer Milenio. Av. Siglo XXI Ciudad Satélite Morelos, Aguascalientes, Ags. México 014499776275 al 79 Fax: 014499776280 Correo electrónico: pilargh@hotmail.com 


\section{Introducción}

El intento suicida es definido por la Organización Mundial de la Salud (OMS) como "un acto con una consecuencia no fatal en la cual el individuo realiza deliberadamente una conducta no habitual con amenaza de muerte, que sin la intervención de otros le causará auto daño, o ingiere una sustancia superior a la dosis terapéuticas generalmente reconocidas y cuyo objetivo es producir cambios que él o ella desean a través de las consecuencias físicas y psíquicas reales o esperadas cercanas a la muerte" ${ }^{1}$. El término suicidio posee en sí mismo un matiz de violencia y agresividad, porque se trata de una voz formada por analogía con el homicidio². Cuando se aborda cualquier área sobre el estudio de los pacientes con intento de suicidio, no hay organismo que tenga información veraz sobre este problema, ya que no es obligatorio reportarlo, mucho menos el reportar la ideación o los planes suicidas. La mortalidad por suicidio ha aumentado lenta pero constantemente en los últimos 40 años. Aunque se observa el incremento del suicidio en hombres y mujeres, el crecimiento es mayor en hombres ${ }^{3}$. El suicidio se encuentra entre el segundo y tercer lugar como causa de muerte en jóvenes de 15 a 19 años de edad y su incidencia se ha triplicado en los países occidentales en los últimos 30 años, especialmente en adolescentes y adultos jóvenes ${ }^{4}$. Por lo general, el $25 \%$ de los pacientes que intentan una vez el suicidio, lo intentarán nuevamente dentro del siguiente año y $10 \%$ lo logrará en un plazo de 10 años. Asimismo, entre 45 y $70 \%$ de quienes intentan el suicidio sufren principalmente de depresión, con rasgos de impulsividad y agresividad y trastornos de la personalidad y alcoholismo, que a menudo van unidos a una pérdida reciente. En el género masculino, el ahorcamiento es el método más usado para el suicidio; en el género femenino predomina la ingesta de grandes dosis de benzodiacepinas, barbitúricos, analgésicos y antibióticos, principalmente ${ }^{5}$. Se han encontrado muchos factores de riesgo para los intentos de suicidio, entre ellos se encuentran factores psicosociales como: pérdida reciente, pérdida de padres en la infancia, inestabilidad familiar, rasgos de personalidad (impulsividad, agresividad), historia familiar de trastornos psiquiátricos (depresión, esquizofrenia, ansiedad, alcoholismo), aislamiento social, desesperanza, estado civil, historia previa de hospitalización por autolesiones, tratamiento psiquiátrico anterior, género masculino, drogadicción, trastorno de personalidad antisocial, intento de suicidio previo, enfermedades discapacitantes, dolorosas, terminales e incapacidad para afrontar y resolver problemas ${ }^{2-4,6-8}$. Es imperioso identificar los grupos en riesgo, para atenderlos en forma adecuada, disminuir el sufrimiento en el paciente y sus familiares, disminuir los costos económicos y de vidas, sin dejar de considerar que cualquier conducta suicida no mortal es potencialmente letal ${ }^{2}$.

La finalidad del presente estudio fue establecer la prevalencia, las características sociodemográficas y psicosociales en pacientes hospitalizados por intento suicida en el servicio de salud mental en un hospital de segundo nivel de atención del estado de Aguascalientes, México. 


\section{Material y métodos}

Se realizó un estudio observacional, transversal, descriptivo y retrospectivo; con 94 pacientes consecutivos de enero a diciembre de 2011; hospitalizados en el servicio de salud mental del Hospital General Tercer Milenio, por presentar intento de suicidio de cualquier edad y sexo, se excluyeron aquellos expedientes incompletos y no hubo eliminados. Se recabaron las variables del estudio y se vaciaron en una cédula de recolección de datos catalogados en cuatro dominios: características sociodemográfi- cas, antecedentes personales patológicos, antecedentes suicidas y motivos del intento suicida actual.

Para el análisis estadístico, se utilizaron medidas de tendencia central, como promedio, desviación estándar, mínimo y máximo para las variables cuantitativas, así como frecuencias simples y porcentajes para las cualitativas; se utilizó la prueba $\times 2$ para la comparación entre variables del estudio mediante el paquete estadístico SPSS versión 15.0 para Windows.

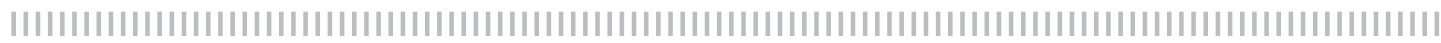

\section{Resultados}

La prevalencia del intento suicida en pacientes hospitalizados en el servicio de Salud Mental fue de 94 pacientes, que representan el $38.8 \%$ de los pacientes hospitalizados en este servicio y el $2.1 \%$ del total de hospitalizados en el Hospital General Tercer Milenio de enero a diciembre del 2011.

La edad media de los pacientes fue de 25.4 11.1 años, con un mínimo de 11 y máximo de 58 años. En cuanto al género predominó el femenino con 63\% (59 pacientes). El rango de edad mas frecuente fue entre 10 a 25 años con $61 \%$ (58 pacientes). El mes donde más se realizaron intentos de suicidio fue en junio con $18 \%$, seguido de enero y mayo con $10 \%$ cada uno. Las ocupaciones se distribuyeron en hogar, desempleado y estudiante con $30 \%$, $29 \%$ y $23 \%$ respectivamente. Con respecto al estado civil, $67 \%$ no tenían pareja (solteros, viudos, divorciado), 33\% tenían pareja estable (casados y unión libre) y el 30\% de los pacientes tenían hijos. La escolaridad predominante fue secundaria con $41 \%$, seguido de primaria con $31 \%$.

El $42 \%$ de los pacientes, recibían atención médica con tratamiento psiquiátrico, usando principalmente benzodiacepinas y antidepresivos. 29\% tenían antecedente de cutting, el $45 \%$ de los pacientes presentaban un intento de suicidio previo, el promedio de intentos suicidas previos, fue de $2.1 \pm 1.8$ intentos, con mínimo de uno y máximo de 9 intentos, encontrándose como mecanismo predominante de lesión durante los intentos de suicidio previos la intoxicación medicamentosa en 59\% (figura 1), de éstos el $61 \%$ usaron benzodiacepinas para la realización del intento. En cuanto al mecanismo de lesión actual, la intoxicación medicamentosa se presentó en $69 \%$ y el ahorcamiento en $15 \%$ (figura 2). El medicamento más usado para el intento actual fueron las benzodiacepinas en $49 \%$ (figura 3 ).

El motivo de realización del acto suicida fue secundario a problemas con su pareja (esposo/novio) en el $28 \%$, seguido de problemas familiares $(21 \%)$. Las comorbilidades encontradas fueron trastorno depresivo en $20 \%$, enfermedades crónico degenerativas $15 \%$, toxicomanías en $11 \%$, aunque en un $25 \%$ no presentaban enfermedades diagnosticadas previamente. Se encontró que el $25 \%$ de los pacientes presentaban uso de tabaquismo, 44\% uso de alcohol y $21 \%$ uso de drogas.

El mecanismo de lesión más frecuente, según el sexo, fue la intoxicación medicamentosa para el femenino y el ahorcamiento en el masculino $(p<0.001)$; en las mujeres el uso de benzodiacepinas fue el más utilizado $(p<0.008)$. (tabla 1$)$ 
No hubo diferencias estadísticas entre el género y tipo de tratamiento psiquiátrico; entre el rango de edad comparado con el mecanismo de lesión y el tipo de medicación actual; así como la comparación entre el antecedente de cutting y el uso de alcohol y drogas $(p>0.05)$.
Se encontró una relación significativa $(\mathrm{p}<$ 0.001) entre la intoxicación por medicamentos y la presencia de antecedente de trastorno depresivo; así como con la presencia de enfermedades crónicas degenerativas.

\section{Tabla I}

Distribución de algunas características psicosociales de acuerdo al sexo

\begin{tabular}{|c|c|c|}
\hline & Mujeres $n=59$ Pacientes & Hombres $n=35$ Pacientes \\
\hline \multicolumn{3}{|l|}{ Estado civil } \\
\hline Sin pareja estable & 37 & 10 \\
\hline Con pareja estable & 22 & 25 \\
\hline \multicolumn{3}{|l|}{ Ocupación } \\
\hline Hogar & 27 & 1 \\
\hline Desempleado & 11 & 16 \\
\hline Estudiante & 14 & 8 \\
\hline Otro & 7 & 10 \\
\hline Tratamiento psiquiátrico previo & 28 & 11 \\
\hline Intento suicidio previo & 31 & 12 \\
\hline Cutting & 19 & 8 \\
\hline \multicolumn{3}{|l|}{ Intento suicidio Actual } \\
\hline Intox. Medicamentos & 49 & 16 \\
\hline Uso de BZD & 23 & 9 \\
\hline Ahorcamiento & 3 & 11 \\
\hline
\end{tabular}

\section{Mecanismo de intento de suicidio previo}

Intoxicación medicamentosa

Lesión/herida

Ahorcamiento

Mecanismos combinados

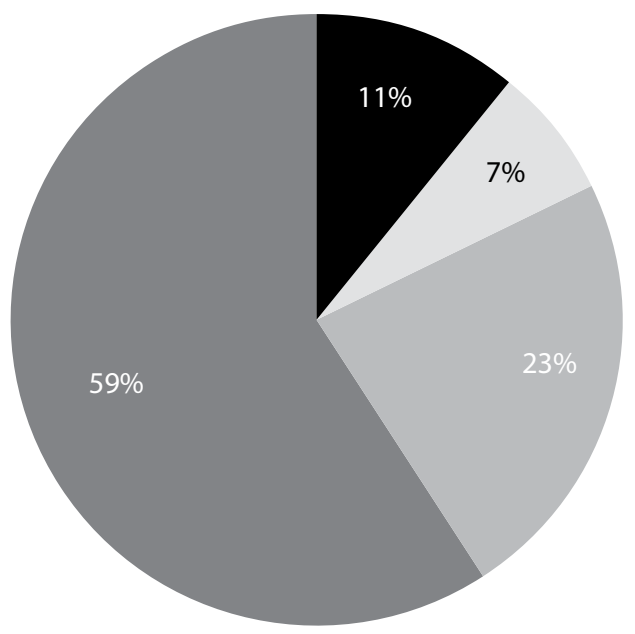




\section{Mecanismo de lesión actual}

Lesión

Ahorcamiento

Intox y otros

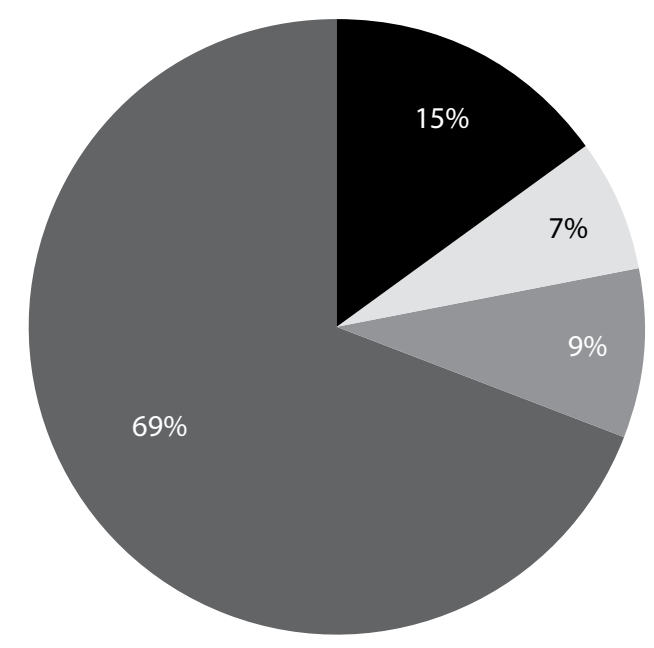

\section{Fármaco usado}

Berizodiaceipinas

Antispicoticos

Anticonvulsivantes

Otros

Se desconoce

Combinación

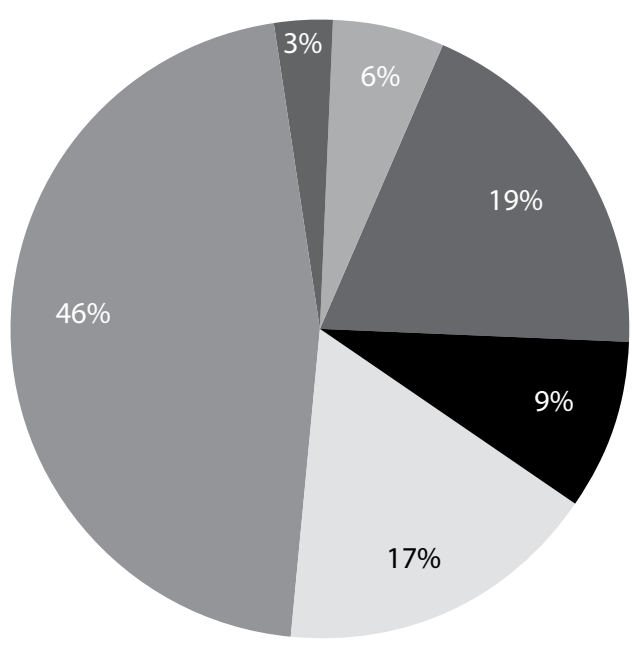

\section{Discusión}

La presencia del intento de suicidio en el servicio de salud mental es "alta" (38.8\%), pero al realizar el porcentaje sobre el total de pacientes hospitalizados se observa "bajo" (2.1\%). Estos valores son muy subjetivos dado que dependen de la demanda, utilización de servicios, la capacidad de ins- talación y recursos del hospital, entre otros factores.

El intento de suicidio fue más frecuente en el sexo femenino con $63 \%$, lo similar a lo reportado por otros estudios por Castro y colaboradores ${ }^{1}$, Coronado-Santos y cols ${ }^{4}$ INEGI $^{9}$. La edad más frecuente encontrada fue entre 10 a 25 años, lo que ha sido re- 
portado en diversos artículos, aunque los rangos han variado entre 10 a 30 años $^{10}$.

Aunque no hubo relación estadísticamente significativa, se presentó mayor frecuencia de intentos suicidas en el mes de junio, como se muestra en otros estudios donde han encontrado mayor frecuencia en verano (junio-septiembre), principalmente en agosto ${ }^{4,6}$.

En ocasiones, la admisión se lleva a cabo contra la voluntad del paciente. En los pacientes con intento suicida, en particular, es de suma importancia decidir si un paciente está en la necesidad de ingreso hospitalario para su estabilización y valoración, dado que después de un intento de suicidio, aproximadamente la mitad de los pacientes tienen que ser hospitalizados sin su autorización ${ }^{11}$.

Los indicadores mundiales revelan que el suicidio es una de las principales causas de muerte en población de 15 a19 años ${ }^{12}$, por lo que se deben realizar medidas de prevención que proporcionen ayuda especializada para el paciente y el entorno familiar. Llama la atención el aumento en el número de casos de intento de suicidio de predominio urbano, por lo que han de tomarse en cuenta algunos factores que han generado un deterioro de las condiciones de vida urbana como el hacinamiento, la falta de empleo y la violencia, entre otros factores ${ }^{8}$.

Nuestros resultados muestran que el no tener pareja estable, es un dato en dos de cada tres pacientes con intento suicida, resultado que es similar a lo publicado por Villafaña y col $^{13}{ }^{13}$, Castro-Obregón ${ }^{1}$ y Vencesla-Moriana ${ }^{6}$.

Es importante resaltar que $45 \%$ de los pacientes presentaban intento de suicidio previo, con 9 intentos en el mayor número de ellos, lo que varía según diversas publicaciones con $13 \%$ en Alba-Aguilar ${ }^{10}$, 13.4\% Castro y Obregón 1 .

El mecanismo de mayor predominio fue la intoxicación medicamentosa $(p<0.001)$ en sexo femenino, igual a lo reportado en 2004 por Coronado-Díaz ${ }^{4}$, 2002 Villafaña y col${ }^{13}$, Garrido y coll4; encontramos mayor uso de benzodiacepinas, en otros estudios se han encontrado este tipo de fármacos para uso frecuente de tentativa suicida ${ }^{14}$.

Existe relevancia en el antecedente de tratamiento psiquiátrico y además uso de benzodiacepinas, lo que nos hace pensar que dicho tratamiento resultó insuficiente o ineficaz en este tipo de pacientes; por lo que debemos retomar las pautas terapéuticas de pacientes con factores de riesgo suicidas, así como reforzar la prevención en nuestra población.

Se han reportado diversos motivos para la realización de tentativa suicida, nosotros encontramos entre los principales: problemas con pareja y familiares, similar a lo reportado en 2002 por Vancesla-Moriana ${ }^{4}$, en 2010 por Alba y Cortez ${ }^{6}$, Villafaña y cols. en 2002, Garrido y cols. $2000^{14}$. Consideramos necesario valorar el entorno familiar del paciente es importante para realizar intervenciones oportunas.

Aunque uno esperaría a más pacientes con trastorno de la personalidad ${ }^{15}$, éste ha sido diagnosticado en sólo unos pocos pacientes. Esto probablemente no refleja la prevalencia real, pero en el transcurso de una sola consulta de emergencia, a menudo es imposible determinar este diagnóstico.

Se ha reportado una comorbilidad en el $20 \%$ de los pacientes, difiriendo con otro estudio que refiere un alta frecuencia en la comorbilidad psiquiátrica en las personas que tratan de suicidarse ${ }^{16}$. En rutina clínica, los psiquiatras probablemente tienden a centrarse en el diagnóstico más directamente relacionado con el intento de suicidio. Sin embargo, información sobre la dependencia de alcohol o trastorno de personalidad como enfermedades concomitantes, resultan torales en la decisión del tratamiento. Los trastornos afectivos son los que mayor prevalencia se encuentran 
en la población general, encontrándose en nuestro estudio como patología predominante en un $20 \%$ de los pacientes, seguido de enfermedades crónicas similar a lo reportando en 2006 por Gutiérrez y cols ${ }^{5}$, Garrido y cols. en $2000^{14}$ y Grua y cols. en $2007^{17}$.

El consumo de alcohol y drogas se han mencionado en diversas publicaciones como un factor de riesgo para llevar a cabo un intento de suicidio ${ }^{18}$, en nuestros resultados se reportó el antecedente de uso de alcohol en $44 \%$ y drogas en $21 \%$.

El tratamiento ambulatorio ha demostrado ser superior al tratamiento de pacientes hospitalizados, en cuanto a la reducción de los síntomas y la satisfacción del paciente; por ello, es conveniente ofrecer al paciente estabilizado en hospital la necesidad de continuar su tratamiento ambulatorio a su egreso hospitalario.

Es imperiosa la necesidad de continuar con esta línea de investigación, bajo estudios causales, tratamientos preventivos eficientes y con ello, realizar una campaña de concientización y prevención de este tipo de problemas que puedan culminar con un suicidio consumado. Además de orientar la identificación de las población mas vulnerables promoviendo acciones de atención oportuna y seguimiento apropiado.

\section{Conclusiones}

La frecuencia de intentos suicidas fue de 94, que representa una prevalencia del $38.8 \%$ en los pacientes hospitalizados en el servicio de salud mental del HGTM; y el $2.1 \%$ del total de hospitalizados.

Los factores psicosociales de los pacientes con intento de suicidio son muy similares en diversas publicaciones, existe una alta frecuencia de intentos de suicidio previos; además de la intoxicación medicamentosa con el uso de las benzodiacepinas como el grupo de medicamentos que con mayor frecuencia se emplean para tal fin.

\section{Bibliografía}

1. Castro PP. Orbegoso RO. Rosales TP. Caracterización del paciente con intento de suicidio en un hospital general de Lima. Rev. perú. med. exp. salud publica, oct./dic. 2006,23(4):293-296.

2. Mosquera F. El comportamiento suicida. Suicidio - Neurobiología, factores de riesgo y prevención. Disponible en: http://es.scribd.com/doc/38536504/28122767sucidio-tellez-forero

3. Borges G. Orozco R. Benjet C. Medina-Mora ME. Suicide and suicidal behaviors in Mexico: Retrospective and current status. Salud Pública Mex 2010; 52:292304.

4. Coronado-Santos S. Díaz-Cervera I. Vargas-Ancona L. Rosado-Franco A. Zapata-Peraza A. Díaz-Talavera S. Prevalencia del intento de suicidio en el Servicio de Urgencias del Hospital General “Dr. Agustín
O'Horán", de enero de 1998 a diciembre de 2003. Rev Biomed 2004;15:207-213.

5. Gutiérrez-García AG. Contreras CM. Orozco-Rodríguez R. El Suicidio, Conceptos Actuales, Salud Mental 2006: 29(5):66-74.

6. Venceslá Martínez J F. Moriana Elvira J A. Conducta autolítica y parasuicida. Características socio demográficas en población infantojuvenil de ámbito rural. Rev Asoc Esp Neuropsiq 2002; XXI (84): 49-64

7. Muñoz MJ. Pinto MV. Callata CH. Napa DN. Perales C A. Ideación suicida y cohesión familiar en estudiantes preuniversitarios entre 15 y 24 años, Lima 2005. Revista Peruana de Medicina Experimental y Salud Pública 2006; 23(004): 239-246.

8.- Caycedo A. Arenas ML. Benítez M. Cavanzo P. Leal G. Guzmán Y. Psycho-social and Family Characte- 
ristics Related to Attempted Suicide in an Adolescent Population in Bogotá-2009. Personal y Bioética 2010;14(2):205-213

9. INEGI. Estadísticas de Intentos de Suicidio y Suicidios de los Estados Unidos Mexicanos 2005.

10. Alba Cortés A. Aguilar Valdés J. Medina Suárez J. Toledo Prado JL. Echemendía B. Causas y factores asociados con el intento de suicidio en adolescentes en la Provincia Sancti Spíritus. Revista Cubana de Higiene y Epidemiología 2010; 48(1):14-22.

11. Hepp U. Moergeli H. Trier SN. Milos G. Schnyder U. Attempted Suicide: Factors Leading to Hospitalization. Can J Psychiatry 2004;49:736-742.

12. Blitz CL. Solomon PL. Feinberg M. Establishing a new research agenda for studying psychiatric emergency room treatment decisions. Ment Health Serv Res 2001;3(1):25-30

13. Villafaña Montiel LG. Gurrola Peña G M. Balcàzar Nava P. Factores psicosociales y predisponentes en el intento de suicidio con pacientes hospitalizados 2002. Psiquiatria.com; 6(4): Disponible en: http:// www.psiquiatria.com/articulos/psiq_general_y_ otras_areas/urgencias_psiq/11699/
14. Garrido Romero R. García García J J. Carballo Ruano E. Luaces Cubells C. Alda Díez J A. Pou Fernández J. Intoxicaciones voluntarias como intento de suicidio. Anales Españoles de Pediatría 2003; 53 (3):213216.

15. Cochrane-Brink KA. Lofchy JS. Sakinofsky I. Clinical rating scales in suicide risk assessment. Gen Hosp Psychiatry 2000;22:445-512.

16. Suominen K. Henriksson M. Suokas J. Isometsa E. Ostamo A. Lonnqvist J. Mental disorders and comorbidity in attempted suicide. Acta Psychiatr Scand 1996;94:234-40.

17. Grau A. Comas P. Suñer R. Peláez E. Sala L. Planas M. Evolución de la ansiedad y depresión detectada durante la hospitalización en un servicio de Medicina Interna. An. Med. Interna 2007; 24: 212-216.

18. Jiménez Saab N G. Lozano Nuevo J J. Rodríguez López L. Vargas Ayala G. Rubio Guerra A. López Islas I. Consumo de alcohol y drogas como factor de riesgo de intento suicida. Med Int. Mex 2005; 21:183-7. 